\title{
ON THE DIRICHLET PROBLEM IN THE AXIOMATIC THEORY OF HARMONIC FUNCTIONS
}

\author{
N. BOBOC, C. CONSTANTINESCU and A. CORNEA
}

In the frame of the recent axiomatic theories of harmonic functions [2], [3], [1], it has been shown that the continuous bounded functions on the boundaries of relatively compact open sets are resolutive [5], [1]. The aim of the present paper is to substitute in these results the continuous functions by Borel-measurable functions and to leave out the restriction that the open sets are relatively compact. $H$. Bauer has replaced the axiom 3 of Brelot's axiomatic by two weaker axioms: the axiom of separation (Trennungsaxiom) and the axiom $K_{1}$. Since the axiom of separation is not fulfilled in some important cases (e.g. the compact Riemann surfaces) we shall weaken this axiom too, substituting it by one of its consequences: the minimum principle for hyperharmonic functions.*)

0. Notations and terminology. We shall use the following notations and terms. A real (resp. numerical) function is a map in the real axis (resp. real axis completed with the points $+\infty,-\infty)$. For a topological space $T$ we denote by $\mathscr{C}(T)$ the set of real continuous functions on $T$. If $U$ is an open set in a topological space $T, \partial_{T} U=\partial U$ (resp. $\bar{U}^{T}=\bar{U}$ ) will stand for the boundary (resp. for the closure) of $U$ in $T$. For a locally compact but noncompact space $T$ we shall denote by $\mathfrak{U}_{T}$ the filter of sets with relatively compact complements.

1. The axioms. Let $X$ be a locally compact Hausdorff space and $\mathscr{N} a$ sheaf on $X$ of real vector spaces of real continuous functions called harmonic functions. We shall suppose that $\mathscr{H}$ will satisfy the axioms $\mathbf{H}_{1}, \mathbf{H}_{2}, \mathbf{H}_{3}$ stated in this paragraph.

Let $U$ be an open set of $X$. An open relatively compact set of $X$ is called regular in $U$ if:

Received March 28, 1963.

*) A similar axiom has been proposed by M. Brelot [4]. 
a) $\bar{V} \subset U$ and $\partial V$ is not empty;

b) for any $f \in \mathscr{C}(\partial V)$ there exists a unique continuous extension on $\bar{V}$ whose restriction to $V$, denoted by $H_{f}^{V}=H_{f}$, is harmonic;

c) from $f \in \mathscr{C}(\partial V)$ and $f \geq 0$ it follows $H_{f}^{V} \geq 0$.

We shall say simply regular instead of regular in $X$.

Let $V$ be regular and $x \in V$. The map $f \rightarrow H_{f}^{V}(x)$ is a positive linear functional on $\mathscr{C}(\partial V)$ and so, there exists a Radon measure $\omega_{x}^{V}$ on $\partial V$, called the harmonic measure of $\boldsymbol{V}$ at the point $\boldsymbol{x}$, such that for any $f \in \mathscr{C}(\partial V)$

$$
H_{f}^{V}(x)=\int f d \omega_{x}^{V}
$$

Axiom $H_{1}$. The regular sets form a basis of $X$.

A point $x \in X$ will be called a zero-point if any harmonic function on $a$ neighbourhood of $x$ vanishes at $x$; we shall denote by $X_{0}$ the set of zero-points.

THEOREM 1. $X_{0}$ is closed, nowhere dense and totally disconnected.

Let $x \in \bar{X}_{0}$ and let $u$ be a harmonic function defined on a neighbourhood $U$ of $x$. Since $u$ vanishes at the points of $X_{0} \cap U$ it vanishes also at $x$. Hence $x \in X_{0}$ and $X_{0}$ is closed.

Let $x \in X_{0}$ and $V$ be a regular neighbourhood of $x$. Then

$$
\left\{y \in V \mid H_{1}^{V}(y)<\frac{1}{2}\right\}
$$

is an open neighbourhood of $x$ contained in $V$ whose boundary does not intersect $X_{0} . \quad X_{0}$ is therefore nowhere dense and totally disconnected.

A numerical function on an open non-empty set $U$ is called hyperharmonic on $U$ if :

a) it does not take the value - -

b) it is lower semi-continuous;

c) for any point $x \in U$ there exists a neighbourhood $U_{s}(x) \subset U$ of $x$, such that for any regular set $V$ in $U_{s}(x)$ and any $y \in V$

$$
s(y) \geq \int^{*} s d \omega_{y}^{v}
$$

A numerical function $s$ on $U$ is called hypoharmonic on $U$ if $-s$ is hyperharmonic on $U$. 
The harmonic functions are hyperharmonic and a function which is simultaneously hyperharmonic and hypoharmonic is harmonic. The sum and the minimum of two hyperharmonic functions and the product of a positive real number with a hyperharmonic function is also hyperharmonic. The hyperharmonic functions form a sheaf on $X$.

We shall say that the minimum principle is valid on an open non-empty set $U$ if one of the following conditions is fulfilled:

a) $U$ is compact and all hyperharmonic functions on $U$ are non-negative;

b) $U$ is non-compact and any hyperharmonic function $s$ on $U$ for which

$$
\lim \inf _{\mathfrak{A}_{U}} s \geq 0
$$

is non-negative.

The open non-empty sets on which the minimum principle is valid will be called M. P.-sets.

TheOREM 2. Let $U, U^{\prime}$ be open non-empty sets, $U^{\prime} \subset U$, and $s$ (resp. $\left.s^{\prime}\right)$ be a hyperharmonic function on $U$ (resp. $\left.U^{\prime}\right)$. We suppose that the function $s^{*}$, defined on $U$ equal to $s$ on $U-U^{\prime}$ and equal to min $\left(s, s^{\prime}\right)$ on $U^{\prime}$, is lower semi-continuous. If any point $x \in U \cap \partial U^{\prime}$ possesses a neighbourhood $U_{x}$ such that either $s \leq s^{\prime}$ on $U_{x} \cap U^{\prime}$ or $U_{x} \cap U^{\prime}$ is an M.P.-set, then $s^{*}$ is a hyperharmonic function.

The conditions a) and b) of the definition of the hyperharmonic functions are satisfied trivially by $s^{*}$ and the same is true for the condition $c$ ) at the points of $U-\partial U^{\prime}$. Let $x$ be a point of $U \cap \partial U^{\prime}$ and $U_{x}$ be a neighbourhood which satisfies the condition from the statement. There exists a neighbourhood $W$ of $x, W \subset U_{x}$, which satisfies the condition c) for $s$. We shall prove that $W$ fulfils the condition c) also for $s^{*}$. This is trivial if $s \leq s^{\prime}$ on $U_{x} \cap U^{\prime}$ since then $s^{*}=s$ on $W$. On the contrary case let $V$ be a regular set in $W$ and $f \in \mathscr{C}(\partial V), f<s^{*}$. The function $s^{*}-H_{f}^{V}$ is lower semi-continuous on $V$, nonnegative on $V-U^{\prime}$, hyperharmonic on $V \cap U^{\prime}$, and

$$
\lim _{V \ni y \rightarrow z} \inf _{y}\left(s^{*}(y)-H_{f}^{V}(y)\right)>0
$$

for any $z \in \partial V$. The function $s_{0}$ defined on $U_{x} \cap U^{\prime}$ equal to 0 on $\left(U_{x} \cap U^{\prime}\right)-V$ and equal to $\min \left(s^{*}-H_{f}^{V}, 0\right)$ on $V \cap U^{\prime}$ is hyperharmonic by the above proof, 
where $U_{x} \cap U^{\prime}$ (resp. $\left.V \cap U^{\prime}\right)$ replaces $U$ (resp. $U^{\prime}$ ) and 0 (resp. $s^{*}-H_{f}^{\prime}$ ) replaces $s$ (resp. $s^{\prime}$ ). If $U_{x} \cap U^{\prime}$ is non-compact then it can be easily verified that

$$
\lim \inf _{\mathscr{A}_{U_{x} \cap U^{\prime}}} s_{0} \geq 0 .
$$

Since $U_{x} \cap U^{\prime}$ is an M.P.-set we get $s_{0} \geq 0, s^{*} \geq H_{f}^{V}$ on $V$. $f$ being arbitrary we get for any $y \in V$

$$
s^{*}(y) \geq \int^{*} s^{*} d \omega_{y}^{V}
$$

Corollary 1. Let $U$ be an M.P.-set and $U^{\prime}$ be an open non-empty subset of $U$. If any point $x \in U \cap \partial U^{\prime}$ possesses a neighbourhood $U_{x}$ such that either there exists a finite hyperharmonic function $s_{x}$ on $U_{x} \cap U^{\prime}$ with inf $s^{*}>0$ or $U_{x} \cap U^{\prime}$ is an M.P.-set, then $U^{\prime}$ is an M.P.-set. The intersection of any regular set with $U$ is an M.P.-set.

Let $s^{\prime}$ be a hyperharmonic function on $U^{\prime}$ such that if $U^{\prime}$ is non-compact

$$
\lim \inf _{\mathfrak{A}_{V}}, s^{\prime} \geq 0
$$

We suppose firstly that there exists a finite hyperharmonic function $s_{0}$ on $U^{\prime}$ with inf $s_{0}>0$. Let $\varepsilon$ be a positive number and $s$ denote the function on $U$ equal to 0 on $U-U^{\prime}$ and equal to $\min \left(s^{\prime}+\varepsilon s_{0}, 0\right)$ on $U^{\prime}$. From the theorem it follows that $s$ is hyperharmonic. If $U$ is non-compact it can be verified easily that

$$
\lim \inf _{\mathfrak{A}_{U}} s \geq 0 \text {. }
$$

Since $U$ is an M.P.-set we get $s \geq 0, s^{\prime} \geq-\varepsilon s_{0}$. $\quad \varepsilon$ being arbitrary and $s_{0}$ finite it follows $s^{\prime} \geq 0 ; U^{\prime}$ is therefore an M.P.-set.

From this proof we see that for any point $x \in U \cap \partial U^{\prime}$ the set $U_{x} \cap U^{\prime}$ is an M.P.-set. Let $s^{*}$ be the function on $U$ equal to 0 on $U-U^{\prime}$ and equal to $\min \left(s^{\prime}, 0\right)$ on $U^{\prime}$. From the theorem it follows that $s^{*}$ is hyperharmonic. If $U$ is non-compact it can be verified easily that

$$
\lim \inf _{\mathfrak{P}_{U}} s^{*} \geq 0 .
$$

Since $U$ is an M.P.-set we get $s^{*} \geq 0, s^{\prime} \geq 0 ; U^{\prime}$ is therefore an M.P.-set.

If $V$ is a regular set, the set $U^{\prime}=V \cap U$ fulfils the required conditions taking $U_{x}=U-\left\{y \in V \mid H_{1}^{V} \leq \frac{1}{2}\right\}$ and $s_{x}=H_{1}^{V}$ for any $x \in U \cap \partial U^{\prime}=U \cap \partial V$. 
DIRICHLET PROBLEM IN THE AXIOMATIC THEORY ÓF HARMONIC FUNCTIONS 77

Corollary 2. Let $U$ be an open non-empty set, s be a hyperharmonic function on $U$ and $V$ be a regular set in $U$. If $V$ is an M.P.-set then the function $s^{V}$ defined on $U$ equal to $s$ on $U-V$ and equal to

$$
x \rightarrow \int^{*} s d \omega_{x}^{V}
$$

on $V$, is hyperharmonic and not greater than s.

Let $f \in \mathscr{C}(\partial V), f \leq s$. Since $V$ is an M.P.-set $s \geq H_{f}^{\mathrm{r}}$ on $V$

$$
s \geq \sup _{f} H_{f}^{V}=s^{V} .
$$

Being on $\bar{V}$ the least upper bound of a family of continuous functions, $s^{v}$ is lower semi-continuous on $\bar{V}$. It follows immediately that $s^{v}$ is lower semicontinuous on $U$. In order to show that $s^{V}$ is hyperharmonic on $V$ let us take a regular set $V^{\prime}$ in $V$. We have for any $x \in V^{\prime}$ and $f \in \mathscr{C}(\partial V), f \leq s$,

$$
s^{V}(x) \geq H_{f}^{V}(x)=\int H_{f}^{V} d \omega_{x}^{V \prime}, \quad s^{V}(x) \geq \sup _{f} \int H_{f}^{V} d \omega_{x}^{V^{\prime}}=\int^{*} s^{V} d \omega_{x}^{V^{\prime}} .
$$

Taking $U_{x}=U$ for any $x \in \partial V$ it follows from the theorem that $s^{v}$ is hyperharmonic.

Axiom $\mathbf{H}_{2}$. The M.P.-sets form a covering of $X$.

This axiom is a theorem in Brelot's [2], [3] and Bauer's [1] axiomatic since in these axiomatics all regular sets are M.P.-sets (Theorem 3 (ii) and 4 ([3] part IV) and Korollar of Lemma 2 [1]).

We shall denote by $\mathfrak{B}$ the set of regular M.P.-sets. From $\mathbf{H}_{1}, \mathbf{H}_{2}$ and the corollary 1 it follows that $\mathfrak{B}$ is a basis of $X$. For any open non-empty set $U$ and any hyperharmonic function $s$ on $U$ and $V \in \mathfrak{B}, \bar{V} \subset U$, we see by corollary 2 that $s^{V}$ is hyperharmonic and for any $x \in V$

$$
s(x) \geq \int^{*} s d \omega_{x}^{v}
$$

From this fact it follows that any M.P.-set can be taken instead of $U_{s}(x)$ in the definition of hyperharmonic functions, this means independently of $s$.

TheOREM 3. The least upper bound of an upper directed set of hyperharmonic functions is also hyperharmonic. 
Let $\mathscr{S}$ be such a set of hyperharmonic functions and $s_{0}$ its least upper bound. $s_{0}$ is lower semi-continuous, does not take the value $-\infty$, and for any $V \in \mathfrak{B}$ and $x \in V$ we have

$$
s_{0}(x)=\sup _{s \in \mathscr{\infty}} s(x) \geq \sup _{s \in \mathscr{\varphi}} \int^{*} s d \omega_{x}^{V}=\int^{*} s_{0} d \omega_{x}^{V} .
$$

Axiom $\mathrm{H}_{3}{ }^{*)}$. For any open non-empty set $U$ the least upper bound of any upper directed set of equally bounded harmonic functions on $U$ is harmonic.

An equivalent statement of this axiom is: on any regular set $V$ the function

$$
x \rightarrow \int f d \omega_{x}^{V}
$$

is harmonic on $V$ for any bounded lower semi-continuous function $f$.

Let $U$ be an open non-empty set. A set $\mathscr{S}$ of hyperharmonic functions on $U$ is called a Perron set if it is lower directed and for any $V \in \mathfrak{B}, \bar{V} \subset U$, and $s \in \mathcal{S}$ it follows $s^{V} \in \mathcal{S}$, where $s^{V}$ denotes the function defined in Corollary 2.

Theorem 4. ([1] Satz 11) The greatest lower bound of a locally equally bounded Perron set is harmonic.

Let $u$ denote the greatest lower bound of a locally equally bounded Perron set $\mathcal{S}$ on an open non-empty set $U$ and let $V \in \mathfrak{B}, \bar{V} \subset U$, such that $\mathscr{S}$ is equally bounded on $\bar{V}$. Then $u$ is equal on $U$ to the greatest lower bound of the set $\left\{s^{V} \mid s \in \mathcal{S}\right\}$ and therefore harmonic by $\mathbf{H}_{3}$.

A potential is a non-negative hyperharmonic function for which any hypoharmonic minorant is non-positive. If $\underline{s}$ is a hypoharmonic minorant of $p+s$, where $p$ is a potential and $s$ a hyperharmonic function, then $\underline{s} \leq s$, since $\underline{s}-s$ is a hypoharmonic minorant of $p$. It follows that the sum of a finite number of potentials is again a potential. It follows further that if the sum of a series of potentials is finite it is also a potential. A non-negative locally bounded hyperharmonic function can be set in exactly one way as a sum of a potential and a harmonic function.

Lemma 1. Let $p$ be a locally bounded potential on $X$. There exists for any $x \in X$ a non-negative hyperharmonic function $p_{x}$, finite at $x$, such that for any

*) This axiom was introduced firstly by $\mathrm{H}$. Bauer [1] (Axiom $\mathrm{K}_{1}$ ). 
filter $\widetilde{\vartheta}$ on $X$ with empty adherence for which

$$
\lim \inf _{\mathfrak{F}} p>0
$$

we have

$$
\lim _{\mathfrak{F}} p_{x}=\infty .
$$

Let $\mathscr{S}$ be the smallest set of hyperharmonic functions which contains $p$ and such that for any $s \in \mathscr{S}$ and $V \in \mathfrak{B}, s^{V} \in \mathscr{S}$. From Theorem 4 it follows that the greatest lower bound of $\mathscr{S}$ is a harmonic function. This function vanishes because it is non-negative and not greater than $p$. For any natural number $n$ let $p_{n} \in \mathscr{S}, p_{n}(x)<\frac{1}{2^{n}}$. The function $p_{x}=\sum_{n=1}^{\infty} p_{n}$ fulfils the required condition.

2. The normed Dirichlet problem. Let $U$ be an open set for which $\partial U$ is non-empty and let $f$ be a numerical function on $\partial U$. We denote by $\overline{\mathscr{S}}_{f}^{U}, x=\overline{\mathscr{S}}_{f}^{U}$ (resp $\mathscr{S}_{f}^{U, x}=\mathscr{S}_{f}^{(J}$ ) the set of lower bounded hyperharmonic (resp. upper bounded hypoharmonic) functions $s$, such that

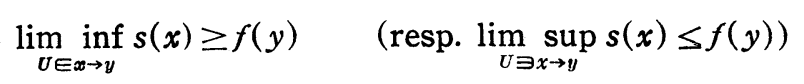

for any $y \in \partial U$ and there exists a compact subset $K_{s}$ of $X$ such that $s \geq 0$ (resp. $s \leq 0)$ on $U-K_{s}$. We denote by $\bar{H}_{f}^{U, x}=\bar{H}_{f}^{U}=\bar{H}_{f}\left(\right.$ resp. $\left.\underline{H}_{f}^{U}, x=\underline{H}_{f}^{U}=\underline{H}_{f}\right)$ the greatest lower bound of $\bar{S}_{f}^{U}$ (resp. the least upper bound of $\mathscr{S}_{f}^{U}$ ). The open set $U$ is called an M.P. -set if $\bar{H}_{0}^{U, x}=0$. Obviously an M.P.-set for which $\partial U$ is non-empty, is an M.P. $P_{0}$-set. From the proof of corollary 1 it results that $U$ is an M.P.-set if and only if for any open relatively compact subset $U^{\prime}$ of $X$ such that $U \cap X_{0} \cap \partial U^{\prime}$ is empty $U^{\prime} \cap U$ is an M.P.-set. If $U$ is an M.P.-set we have $\underline{H}_{f}^{U} \leq \bar{H}_{f}$.

If $\left\{f_{n}\right\}$ is a decreasing sequence such that $\underline{H}_{f_{n}}$ are harmonic then

$$
\lim _{n \rightarrow \infty} \underline{H}_{f_{n}}=\underline{H}_{\lim f_{n}} \text {. }
$$

Indeed let $x$ be a point of $U$ and for any $n, s_{n} \in \mathscr{S}_{f_{n}}^{U}$ such that $s_{n}(x)>$ $\underline{H}_{f_{n}}(x)-\frac{1}{2^{n}}$. The functions

$$
s=\lim _{n \rightarrow \infty} \underline{H}_{f_{n}}, \quad \boldsymbol{s}_{m}^{\prime}=\sum_{n=m}^{\infty}\left(s_{n}-\underline{H}_{f_{n}}\right)
$$

are hypoharmonic (Theorem 3) and $s+s_{m}^{\prime} \in \mathscr{S}_{f_{n}}^{U}$ for any $n$. Hence $s+s_{m}^{\prime} \in \underline{\mathscr{S}}_{n \rightarrow \infty}^{U} f_{n}$, 


$$
\begin{aligned}
& s(x)-\frac{1}{2^{m-1}}<s(x)+s_{m}^{\prime}(x) \leq \underline{H}_{n \rightarrow \infty} f_{n}(x), \\
& s(x) \leq \underline{H}_{n \rightarrow \infty} \lim _{n \rightarrow \infty}(x) \leq \lim _{n \rightarrow \infty} \underline{H}_{f_{n}}(x)=s(x) .
\end{aligned}
$$

We have

$$
\bar{H}_{f_{1}+f_{2}} \leq \bar{H}_{f_{1}}+\bar{H}_{f_{2}}, \quad \underline{H}_{f_{1}+f_{2}} \geq \underline{\underline{H}} \underline{f}_{1}+\underline{H}_{f_{2}},
$$

wherever the right side has a sense, where $f_{1}+f_{2}$ is defined arbitrarily on the set

$$
\left\{y \in \partial U \mid f_{1}(y)=+\infty, f_{2}(y)=-\infty\right\} \cap\left\{y \in \partial U \mid f_{1}(y)=-\infty, f_{2}(y)=+\infty\right\},
$$

and for $\alpha>0$

$$
\bar{H}_{\alpha f}=\alpha \bar{H}_{f}, \quad \underline{H}_{\alpha f}=\alpha \underline{H}_{f}, \quad \bar{H}_{-f}=-\underline{H}_{f} .
$$

If the functions $\bar{H}_{f}, \underline{H}_{f}$ are finite (resp. harmonic) and equal the function $f$ is called resolutive (resp. harmonic resolutive) and

$$
H_{f}^{U}, x=H_{f}^{U}=H_{f}=\bar{H}_{f}=\underline{H}_{f}
$$

is called the normed solution of Dirichlet problem with $f$ as boundary function. If $U$ is an M.P.-set, the set of resolutive (resp. harmonic resolutive) real functions on $\partial U$ form a real vector space. If $f_{1}, f_{2}$ are non-negative harmonic resolutive functions then $\max \left(f_{1}, f_{2}\right)$ is resolutive and $H_{\max \left(f_{1}, f_{2}\right)}$ is the least harmonic majorant of $H_{f_{1}}, H_{f_{2}}$.

Lemma 2. Let $U$ be an open set, $y \in \partial U$ and $₹$ be a filter on $U$ converging to $y$. We suppose that there exists a fundamental system $\mathfrak{B}$ of regular neighbourhoods of $y$ such that for any $V \in \mathfrak{B}$ there exists a non-negative hyperharmonic function $s_{V}$ on $V \cap U$ such that

$$
\lim _{\mathfrak{F}} s_{V}=0
$$

and for any $z \in U \cap \partial V$

$$
\lim _{U \cap V \ni x \rightarrow z} \inf _{V}(x)>0 .
$$

Let $s$ be a hyperharmonic (resp. hypoharmonic) function on the intersection of $U$ with a neighbourhood of $y$ for which

$$
\left.\lim _{U \ni x \rightarrow y} \inf _{x \rightarrow} s(x)=1 \quad \text { (resp. } \lim _{U \ni x \rightarrow y} \sup _{U \rightarrow x} s(x)=1\right) .
$$


DIRICHLET PROBLEM IN THE AXIOMATIC THEORY OF HARMONIC FUNCTIONS 81

Let $f$ be a non-negative numerical function on $\partial U$, for which $\bar{H}_{f}^{U}$ (resp. $\underline{H}_{f}^{U}$ ) is harmonic on $U$ and bounded on a neighbourhood of $y$. Then

$$
\begin{gathered}
\lim \sup _{\mathfrak{F}} \bar{H}_{f}^{U} \leq\left(\lim _{\partial U \ni x \rightarrow y} \sup _{f} f(x)\right)\left(\lim \sup _{\mathfrak{F}} s(x)\right) \\
\left(\text { resp. } \lim \inf _{\mathfrak{F}} \underline{H}_{f}^{U} \geq\left(\lim _{\partial U \ni x \rightarrow y} f(x)\right)\left(\lim \inf _{\mathfrak{F}} s(x)\right)\right)^{*)} .
\end{gathered}
$$

We shall prove this lemma following the proof of Theorem 22 [3] Part IV.

We suppose that

$$
\lim _{\partial I J \exists x \rightarrow y} \sup _{x} f(x)<\infty \quad\left(\text { resp. } \lim _{\partial U \ni x \rightarrow y} \inf _{x \rightarrow y} f(x)>0\right)
$$

since on the contrary case the assertion is trivial. Let $\alpha$ be a positive number

$$
\alpha>\lim _{\partial U \ni x \rightarrow y} \sup _{x} f(x) \quad\left(\text { resp. } \alpha<\lim _{\partial U \ni x \rightarrow u} \inf _{x \rightarrow y} f(x)\right),
$$

$\varepsilon$ be a positive number smaller than 1 , and $V \in \mathfrak{B}$ such that $\bar{H}_{f}^{U}$ (resp. $\underline{H}_{f}^{U}$ ) is bounded on $\bar{V} \cap U, f<\alpha$ (resp. $f>\alpha$ ) on $V \cap \partial U$, and $s>1-\varepsilon$ (resp. $s<1+\varepsilon$ ) on $\bar{V} \cap U$. Let further $K$ be a compact set in $U \cap \partial V$ for which

$$
\omega_{y}^{v}(U \cap \partial V-K)<\varepsilon .
$$

There exists a positive number $\beta$ such that for any $z \in K$

$$
\begin{aligned}
& \lim _{U \cap V^{\prime} \inf _{x \rightarrow z}}\left(\frac{\alpha}{1-\varepsilon} s(x)+\beta s_{V}(x)\right)>\bar{H}_{f}^{U}(z) \\
& \text { (resp. } \left.\lim _{U^{\prime} \cap \sup _{\exists \rightarrow z}}\left(\frac{\alpha}{1+\varepsilon} s(x)-\beta s_{V}(x)\right)<\underline{H}_{f}^{U}(z)\right) .
\end{aligned}
$$

We denote by $h$ the harmonic function on $V$

$$
x \rightarrow \omega_{x}^{V}(U \cap \partial V-K)
$$

by $\gamma$ the number $\sup _{\bar{H}} \bar{H}_{f}^{U}(x)$ (resp. sup $\underline{H}_{f}^{U}(x)$ ), and by $s_{0}$ the function defined

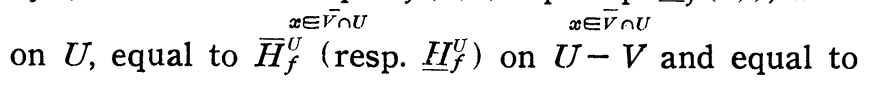

$$
\begin{gathered}
\min \left(\bar{H}_{f}^{U}, \frac{\alpha}{1-\varepsilon} s+\beta s_{V}+\gamma h\right) \\
\left(\text { resp. } \max \left(\underline{H}_{f}^{U}, \frac{\alpha}{1+\varepsilon} s-\beta s_{r^{-}}-(\alpha+\gamma) h\right)\right)
\end{gathered}
$$

on $U \cap V$. From Theorem 2 we see that $s_{0}$ is a hyperharmonic (resp. hypohar-

*) With the convention $0 \cdot \infty=\infty, \infty \cdot 0=0$. 
monic) function. For any $s^{\prime} \in \overline{\mathscr{S}}_{f}^{U}\left(\right.$ resp. $\left.\mathscr{S}_{f}^{U}\right)$ it can be verified that $s^{\prime}-\bar{H}_{f}^{U}+s_{0} \in \overline{\mathscr{S}}_{f}^{U}\left(\right.$ resp. $\left.s^{\prime}-\underline{H}_{f}^{U}+s_{0} \in \underline{\mathscr{S}}_{f}^{U}\right)$. Hence

$$
\begin{aligned}
& s^{\prime}-\bar{H}_{f}^{U}+s_{0} \geq \bar{H}_{f}^{U} \quad\left(\text { resp. } s^{\prime}-\underline{H}_{f}^{U}+s_{0} \leq \underline{H}_{f}^{U}\right), \\
& s_{0} \geq \bar{H}_{f}^{U} \quad\left(\text { resp. } s_{0} \leq \underline{H}_{f}^{U}\right), \\
& \lim \sup _{\mathfrak{F}} \bar{H}_{f}^{U} \leq \lim \sup _{\mathfrak{F}} s_{0} \leq \lim \sup _{\mathfrak{F}}\left(\frac{\alpha}{1-\varepsilon} s+\beta s_{V}+\gamma h\right) \leq \\
& \quad \leq \frac{\alpha}{1-\varepsilon} \lim \sup _{\mathfrak{F}} s+\gamma \varepsilon
\end{aligned}
$$

$\left(\right.$ resp. $\lim \inf _{\mathfrak{F}} \underline{H}_{f}^{U} \geq \lim \inf _{\mathfrak{F}} s_{0} \geq \lim \inf _{\mathfrak{F}}\left(\frac{\alpha}{1+\varepsilon} s-\beta s_{V}-(\alpha+\gamma) h\right)=$

$$
\left.\geq \frac{\alpha}{1+\varepsilon} \lim \inf _{\mathfrak{F}} s-(\alpha+\gamma) \varepsilon\right) \text {. }
$$

$\varepsilon$ and $\alpha$ being arbitrary, we get

$$
\begin{gathered}
\lim \sup _{\mathfrak{F}} \bar{H}_{f}^{U} \leq\left(\lim _{\partial U \ni \rightarrow x} \sup _{x \rightarrow y} f(x)\right)\left(\lim \sup _{\mathfrak{F}} s\right) \\
\left(\text { resp. } \lim \inf _{\mathfrak{F}} \underline{H}_{f}^{U} \geq\left(\lim _{\partial U \ni x \rightarrow y} \inf _{x \rightarrow y} f(x)\right)\left(\lim \inf _{\mathfrak{F}} s\right)\right) .
\end{gathered}
$$

REMARK. If in this lemma $s$ is harmonic and $\lim _{V \exists x \rightarrow J} s(x)=1$, then it can be proved in the same way that

$$
\begin{aligned}
& \lim \sup _{\mathfrak{F}} \bar{H}_{f}^{U} \leq \lim _{\partial U_{\exists} \sup _{y \rightarrow x}} f(x), \\
& \lim \inf _{\mathfrak{F}} \underline{H}_{f}^{U} \geq \lim _{\partial U \exists: x \rightarrow y} \inf _{x \rightarrow y}(x)
\end{aligned}
$$

for any $f$ (not necessarily non-negative) provided that $\bar{H}_{f}^{U}, \underline{H}_{f}^{U}$ are bounded. This remark will be not used in the sequel.

Let $U$ be an open set and $y \in \partial U$. For any non-negative hyperharmonic (resp. hypoharmonic) function $s$, defined on the intersection of $U$ with a neighbourhood of $y$ for which

$$
\lim _{U \exists x \rightarrow y} \inf s(x)=1 \quad\left(\text { resp. } \lim _{U \exists x \rightarrow y} \sup _{x \rightarrow y} s(x)=1\right),
$$

we set

$$
\begin{aligned}
& \sigma_{U}^{*}(y, s)=\lim _{U^{\top} \ni x \rightarrow y} \sup _{x \rightarrow y} s(x) \quad\left(\text { resp. } \sigma_{* v}(y, s)=\lim _{U \ni x \rightarrow y} \inf s(x)\right), \\
& \sigma_{U}^{*}(y)=\sigma^{*}(y)=\inf _{s} \sigma_{U}^{*}(y, s) \quad\left(\text { resp. } \sigma_{* U}(y)=\sigma_{*}(y)=\sup _{s} \sigma_{* U}(y, s)\right) .
\end{aligned}
$$

If there does not exist an $s$ with the required conditions we set $\sigma^{*}(y)=\infty$ (resp. $\sigma_{*}(y)=0$ ). We observe that the existence of a harmonic function on a neighbourhood of $y$ which is different from zero in $y$ implies $\sigma^{*}(y)=\sigma_{*}(y)=1$. 
Hence if $\sigma^{*}(y)>1$ or if $\sigma_{*}(y)<1, y$ is a zero point. The function $\sigma^{*}$ (resp. $\sigma_{*}$ ) is upper (resp. lower) semi-continuous as can be easily verified. We set

$$
A_{U}^{*}=A^{*}=\left\{y \in \partial U \mid \sigma^{*}(y)=1\right\}, \quad A_{* U}=A_{*}=\left\{y \in \partial U \mid \sigma_{*}(y)=1\right\} .
$$

Of course $\partial U-A^{*} \cap A_{*} \subset X_{0}$.

Lemma 3. Let $U$ be an open set with non-empty boundary, $p$ be a locally bounded potential on $U$, positive on a neighbourhood of any point of $\partial U-X_{0}$, and $f$ be a non-negative function on $\partial U$. a) If $f$ is lower semi-continuous and $\underline{H}_{f}$ harmonic on $U$ bounded in a neighbourhood of any boundary point of $U$ then

$$
\bar{H}_{f_{\sigma_{*}}} \leq \underline{H}_{f}^{*)} .
$$

b) If $f$ is upper semi-continuous, $\bar{H}_{f}$ harmonic on $U$ and bounded in a neighbourhood of any boundary point of $U$, and if $U$ is either relatively compact or an M.P. $P_{0 .}$ set then

$$
\bar{H}_{f} \leq \underline{H}_{f_{\sigma^{*}}}{ }^{*} .
$$

Let $x$ be a point of $U$ and $p_{x}$ be the hyperharmonic function associated to $x$ and $p$ by Lemma 1 . Let $\varepsilon$ be a positive number and $y \in \partial U$. We want to prove that

$$
\begin{gathered}
\lim \sup _{U \ni z \rightarrow y}\left(\bar{H}_{f}(z)-\varepsilon p_{x}(z)\right) \leq f(y) \sigma^{*}(y) \\
\text { (resp. } \left.\lim _{U \ni z \rightarrow y}\left(\underline{H}_{f}(z)+\varepsilon p_{x}(z)\right) \geq f(y) \sigma_{*}(y)\right) .
\end{gathered}
$$

Let $y \notin X_{0}$ and $\mathfrak{u}$ be an ultrafilter on $U$ converging to $y$ for which

$$
\begin{gathered}
\lim _{\mathfrak{U}}\left(\bar{H}_{f}-\varepsilon p_{x}\right)=\lim _{U \ni z \rightarrow y} \sup _{U}\left(\bar{H}_{f}(z)-\varepsilon p_{x}(z)\right) \\
\text { (resp. } \left.\left.\lim _{\mathfrak{U}}\left(\underline{H}_{f}+\varepsilon p_{x}\right)=\lim _{U \ni z \rightarrow y} \inf _{f}(z)+\varepsilon p_{x}(z)\right)\right) .
\end{gathered}
$$

If

$$
\lim _{\mathfrak{U}} p \neq 0
$$

then

$$
\lim _{\mathfrak{u}} p_{x}=\infty
$$

and the required inequality is proved. In the opposite case the inequality follows from Lemma 2 taking $\mathfrak{B}$ equal to the set of all regular neighbourhoods

*) With the convention $\infty \cdot 0=0,0 \cdot \infty=\infty$. 
of $y$ and for any $V \in \mathfrak{B}, s_{V}=p$; in the role of the function $s$ we can take here any harmonic function in a neighbourhood of $y$ equal to 1 at $y$. Suppose now $y \in X_{0}$. Then for any regular neighbourhood $V$ of $y$ we have $H_{1}^{V}(y)=0$. Let $s$ be a hyperharmonic (resp. hypoharmonic) function on the intersection of $U$ with a neighbourhood of $y$ with

$$
\left.\lim _{U \ni z \rightarrow y} \inf s(z)=1 \quad \text { (resp. } \lim _{U \ni z \rightarrow y} \sup _{z} s(z)=1\right) .
$$

Taking in Lemma 2, $\mathfrak{B}$ the set of all regular neighbourhoods of $y$, for any $V \in \mathfrak{B}$ $H_{1}^{V^{r}}$ as $s_{V}$, and the trace of $\mathfrak{B}$ on $U$ as $\mathfrak{F}$ we get from this lemma

$$
\begin{gathered}
\lim \sup _{U \Xi z \rightarrow y} \bar{H}_{f}(z) \leq f(y) \sigma^{*}(y, s) \\
\text { (resp. } \left.\lim _{\tau^{*} \exists z \rightarrow y} \underline{\inf }_{f}(z) \geq f(y) \sigma_{*}(y, s)\right) .
\end{gathered}
$$

$s$ being arbitrary the assertion is proved also in this case.

a) $\underline{H}_{f}+\varepsilon p_{x} \in \overline{\mathscr{Y}}_{f_{J_{*}}}^{U}$. Indeed the above proof shows that the condition at the points of $\partial U$ is fulfilled. Since $\underline{H}_{f}, p_{x}$ are non-negative, $\underline{H}_{f}+\varepsilon p_{x}$ is nonnegative on $U$. From this relation we get

$$
\bar{H}_{f \sigma_{*}} \leq \underline{H}_{f}+\varepsilon p_{x} .
$$

$p_{x}$ being finite at $x$ and $\varepsilon$ and $x$ being arbitrary we obtain

$$
\bar{H}_{f \sigma_{*}} \leq \underline{H}_{f} .
$$

b) Suppose now that $U$ is relatively compact. Then from the first part of the proof we have $\bar{H}_{f}-\varepsilon p_{x} \in \mathscr{I}_{f_{\sigma^{*}} \text {, }}^{U}$

$$
\bar{H}_{f}-\varepsilon p_{x} \leq \underline{H}_{f \sigma^{*}} .
$$

$p_{x}$ being finite at $x$ and $\varepsilon$ and $x$ being arbitrary we obtain

$$
\bar{H}_{f} \leq \underline{H}_{f^{*}} .
$$

Suppose now that $U$ is a non-relatively compact M.P. $P_{0}$-set. Let $G$ be a relatively compact open set whose boundary does not intersect $X_{0}$. From the proof of Corollary 1 it follows that $G \cap U$ is an M.P.-set. Let $f_{G}$ be the function defined on $\partial(G \cap U)$ equal to 0 on $\bar{G} \cap \partial U$ and equal to $\bar{H}_{f}^{U}$ on $U \cap \partial G$. Since $U$ is an M.P. $P_{0}$-set $\bar{H}_{f}^{U}$ is non-negative. From $\bar{H}_{f}^{U} \in \overline{\mathscr{S}}_{f G}^{G \cap U}$ and the fact that $G \cap U$ is an M.P. ${ }_{0}$-set we deduce that $\bar{H}_{f_{G}}^{g \cap U}$ is non-negative harmonic and bounded. The 
function $s_{G}$ on $U$ equal to $\bar{H}_{f}^{U}$ on $U-\bar{G}$ equal to $\bar{H}_{f_{G}}^{G \cap U}$ on $G \cap U$ and equal to

$$
\lim _{G \cap U \ni z \rightarrow y} \inf _{H_{G}}^{G \cap U}(z)
$$

at any $y \in U \cap \partial G$ is hyperharmonic. Indeed $s_{G}$ fulfils the conditions a) and $\mathrm{b}$ ) from the definition of hyperharmonic functions. Let $\bar{s} \in \overline{\mathscr{S}}_{f G}^{G \cap U}$. The function $\bar{s}_{G}$ on $U$ equal to $\bar{H}_{f}^{T}$ on $U-G$ and equal to $\min \left(\bar{s}, \bar{H}_{f}^{I T}\right)$ on $G \cap U$ is hyperharmonic by Theorem 2. Let $V$ be a regular set in $U$ and $f \in \mathscr{C}(\partial V), f \leq s_{G}$. We have on $V$

$$
\bar{s}_{G} \geq H_{f}^{V}
$$

From this inequality we get on $V-\partial G$

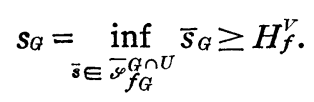

For any $y \in V \cap \partial G$ we have

$$
\boldsymbol{s}_{G}(y)=\lim _{G \cap U \ni z \rightarrow y} \inf _{G}(z) \geq H_{f}^{V}(y) .
$$

$s_{G}$ is therefore hyperharmonic.

Let $\&$ denote the set of open relatively compact sets $G$ for which $X_{0} \cap \partial G=\phi$. By Theorem $1 X_{0}$ is closed and totally disconnected. Hence for any compact set $K$, there exists an open set $G \in \mathbb{B}$ containing $K$. The family $\left(s_{G}\right)_{G \in \mathbb{S}}$ is contained in a Perron set with the same greatest lower bound since from $G_{1}, G_{2}, G \in \mathbb{B}, G \supset \overline{G_{1} \cup G_{2}}$, it follows

$$
s_{G} \leq s_{G_{1}}, \quad s_{G} \leq s_{Q_{2}} .
$$

All functions $s_{G}$ being non-negative and dominated by $\bar{H}_{f}^{U}$ the greatest lower bound $u$ of this family is harmonic by Theorem 4 and non-negative. Let $\eta$ be a positive number, and $y \in \partial U$. Taking a $G \in \mathbb{B}$, which contains $y$ we get from the first part of the proof

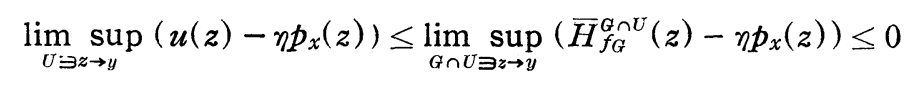

if $y \notin X_{0}$. If $y \in X_{0}$, let $V$ be a regular neighbourhocd of $y, \bar{V} \subset G$, and $\alpha$ a sufficiently great number such that

$$
\bar{H}_{f}^{U}<\alpha
$$

on $\bar{V} \cap U$. We denote by $s_{0}$ the function on $U \cap G$ equal to $\bar{H}_{f}^{U}$ on $U \cap G-V$ 
and equal to $\min \left(\bar{H}_{f}^{U}, \alpha H_{1}^{V}\right)$ on $V \cap U$. From Theorem 2 it follows that $s_{0}$ is hyperharmonic. $s_{0}$ belongs to $\overline{\mathscr{S}}_{f_{G}}^{U \cap G}$ and we get

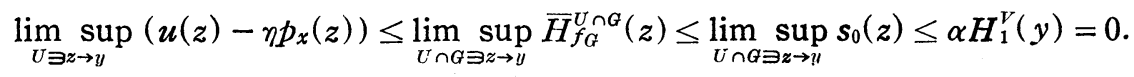

Hence for any $\bar{s} \in \overline{\mathscr{S}}_{f}^{U}$ and $y \in \partial U$ we have

$$
\left.\lim _{U \ni z \rightarrow y} \inf _{(\bar{s}}(z)-u(z)+\eta p_{x}(z)\right) \geq f(y) .
$$

From this inequality and from $\bar{s} \geq u$ it follows $\bar{s}-u+\eta p_{x} \in \overline{\mathscr{S}}_{f}^{U}$,

$$
\bar{s}(x)-u(x)+\eta p_{x}(x) \geq \bar{H}_{f}^{U}(x) .
$$

$\bar{s}$ and $\eta$ being arbitrary and $p_{x}(x)$ finite we deduce $u(x)=0$. Let $\left\{G_{n}\right\}$ be a sequence from $\$$ for which

$$
\sum_{n=0}^{\infty} s_{G_{n}}(x)<\infty
$$

and let us denote by $s_{x}$ the hyperharmonic function

$$
s_{x}=\sum_{n=0}^{\infty} s_{G_{n}} .
$$

It is easy to verify that $\bar{H}_{f}-\varepsilon s_{x}$ is non-positive outside a compact set of $X$. From this and from the first part of the proof it follows

$$
\bar{H}_{f}-\varepsilon p_{x}-\varepsilon s_{x} \in \mathscr{S}_{f \sigma^{*}}^{U}, \quad \bar{H}_{f}-\varepsilon p_{x}-\varepsilon s_{x} \leq \underline{H}_{f \sigma^{*}} .
$$

$p_{x}$ and $s_{x}$ being finite at $x$ and $\varepsilon$ and $x$ being arbitrary we obtain

$$
\bar{H}_{f} \leq \underline{H}_{f \sigma^{*}} .
$$

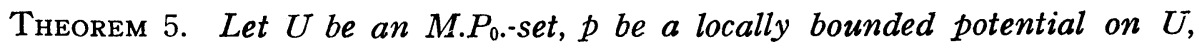
positive on a neighbourhood of any point of $\partial U-X_{0}$, and $s$ be a non-negative hyperharmonic function on $U$ bounded in the neighbourhood of any point of $\bar{U}$ and for which

$$
\lim _{C^{\prime} \exists x \rightarrow y} \inf s(x) \geq 1
$$

for any $y \in A_{*}$. If $f$ is a bounded lower semi-continuous function on $\partial U$, then $f \chi_{A_{*}}$ is harmonic resolutive, where $\chi_{A_{*}}$ is the characteristic function of $\dot{A}_{*}$.

Let us denote

$$
B=\left\{y \in \partial U \mid \lim _{U \ni x \rightarrow y} \inf _{x} s(x)>\frac{1}{2}\right\}
$$


$B$ is an open set on $\partial U$ and $A_{*} \subset B$. Suppose firstly $f \geq 0$. Then, for a suitable positive number $\alpha, \alpha s \in \overline{\mathcal{S}}_{f_{B} \sigma_{*}^{n}}^{T}$, where $\chi_{B}$ is the characteristic function of $B$ and $n$ a natural number. Hence $\underline{H}_{f \times_{B} \sigma_{*}^{n}}$ is harmonic and bounded in a neighbourhood of any boundary point of $U$. We get by the preceding lemma

$$
\bar{H}_{f \chi_{A *}} \leq \bar{H}_{f x_{B} \sigma_{*}^{n+1}} \leq \underline{H}_{f \chi_{B} \sigma_{*}^{n}} .
$$

It follows

$$
\underline{H}_{f x_{A *}} \leq \bar{H}_{f x_{A *}} \leq \lim _{n \rightarrow \infty} \underline{H}_{f x_{B^{\sigma}} \sigma_{*}^{n}}=\underline{H}_{f x_{A *}} .
$$

For a general $f$ denote

$$
\alpha=\inf f \text {. }
$$

The functions $\chi_{A_{*}},(f-\alpha) \chi_{A_{*}}$ being resolutive, the function

$$
f \chi_{A_{*}}=(f-\alpha) \chi_{A_{*}}+\alpha \chi_{A_{*}}
$$

is also resolutive.

If $U$ is an $M P_{0}$. set, a set $M \subset \partial U$ is called negligible if $\bar{H}_{\chi_{M}}=0$, where $\chi_{M}$ denotes the characteristic function of $M . A$ set $M$ is negligible if and only if for any $x \in U$ there exists a non-negative hyperharmonic function $s_{x}$ finite at $x$ for which

$$
\lim _{U \ni z \rightarrow y} \inf _{x}(z)=\infty
$$

for any $y \in M$. The condition is obviously sufficient. If $M$ is negligible there exists for any natural number $n$ an $s_{n} \in \overline{\mathcal{S}}_{\mathrm{x}_{M}}^{V}$ such that

$$
s_{n}(x)<\frac{1}{2^{n}}
$$

and we can take

$$
s_{x}=\sum_{n=1}^{\infty} s_{n}
$$

If two functions $f, g$ on $\partial X$ differ only on a negligible set, then

$$
\underline{H}_{f}=\underline{H}_{g}, \quad \bar{H}_{f}=\bar{H}_{g} .
$$

Indeed we have for $x \in U$ and $\varepsilon>0, \varepsilon s_{x} \in \overline{\mathcal{S}}_{|f-g|}^{(v}$, and therefore

$$
\begin{aligned}
& \bar{H}_{|f-g|}=0, \\
& \bar{H}_{f} \leq \bar{H}_{|f-g|}+\bar{H}_{g}=\bar{H}_{g} .
\end{aligned}
$$


Theorem 6. Let $U$ be an M.P.-set, $p$ be a locally bounded potential on $U$, positive on a neighbourhood of any point of $\partial U-X_{0}$, and $f_{0}$ be a non-negative upper (resp. lower) semi-continuous function on $\partial U$ for which $\bar{H}_{f_{0}}$ (resp. $\left.\underline{H}_{f_{0}}\right)$ is harmonic on $U$ and bounded in a neighbourhood of any boundary point of $U$. If $\partial U-A^{*}$ (resp. $\left.\partial U-A_{*}\right)$ is negligible then any Borel function ${ }^{*)} f,|f| \leq f_{0}$, is harmonic resolutive.

Let $f$ be a non-negative upper (resp. lower) semi-continuous function on $\partial U$, $f \leq f_{0} . \quad \bar{H}_{f}$ (resp. $\underline{H}_{f}$ ) is harmonic and bounded in a neighbourhood of any boundary point of $U$. This is obvious for $\underline{H}_{f}$. Let $\mathscr{S}$ be the smallest Perron set containing the set $\left\{\min \left(\bar{s}, \bar{H}_{f_{0}}\right) \mid \bar{s} \in \overline{\mathscr{S}}_{f_{0}}^{U}\right\}$. For any $s^{\prime} \in \overline{\mathscr{S}}_{f_{0}}^{U}$ and any $s \in \mathcal{S}$ we have $s+s^{\prime}-\bar{H}_{f_{0}}^{U} \in \overline{\mathscr{S}}_{f}^{U}$. Hence

$$
\bar{H}_{f}^{U} \leq s+s^{\prime}-\bar{H}_{f_{0}}^{U}, \quad \bar{H}_{f_{0}}^{U} \leq \inf _{s \in \mathscr{\varphi}} s .
$$

The converse inequality being trivial, $\bar{H}_{f_{0}}^{U}$ is the greatest lower bound of a Perron set and therefore harmonic.

From Lemma 3 and from the fact that $f$ and $f \sigma^{*}$ (resp. $f \sigma_{*}$ ) differ only on a negligible set we have

$$
\begin{gathered}
\underline{H}_{f} \leq \bar{H}_{f} \leq \underline{H}_{f \sigma^{*}}=\bar{H}_{f} \\
\text { (resp. } \left.\underline{H}_{f} \leq \bar{H}_{f}=\bar{H}_{f \sigma_{*}} \leq \underline{H}_{f}\right),
\end{gathered}
$$

and $f$ is resolutive.

Let $\mathfrak{B}$ be the class of Borel-sets $M \subset \partial U$ for which $f_{0} \chi_{M}$ is resolutive. $\mathfrak{B}$ contains the closed (resp. open) sets and from $M \in \mathfrak{B}$ it follows $\partial U-M \in \mathfrak{B}$. Let $M_{1}, M_{2} \in \mathfrak{B}$. From

$$
f_{0} \chi_{M_{1} \cup M_{2}}=\max \left(f_{0} \chi_{M_{1}}, f_{0} \chi_{M_{2}}\right)
$$

it follows that $M_{1} \cup M_{2} \in \mathfrak{B}$. Let $\left\{M_{n}\right\}$ be an increasing sequence of $\mathfrak{B}$. Then $f_{0} \chi_{M_{n}} \uparrow f_{0} \chi_{n=1}^{\infty} M_{n}$ and $\bigcup_{n=1}^{\infty} M_{n} \in \mathfrak{B}$. $\mathfrak{B}$ coincides therefore with the class of all Borel sets.

Let $f$ be a Borel function on $\partial U, 0 \leq f \leq f_{0}$, and $n$ a natural number. For any natural number $i, 0 \leq i \leq 2^{n}$, we design

*) A function is called a Borel function if it is Borel measurable. The class of Borel sets is the smallest class of sets which contains the open sets and is closed with respect to countable union and contians together with a set its complement. 


$$
\begin{aligned}
A_{i} & =\left\{y \in \partial U \mid \frac{i}{2^{n}} f_{0}(y) \leq f(y)<\frac{i+1}{2^{n}} f_{0}(y)\right\} . \\
f_{n} & =f_{0} \sum_{i=0}^{n} \frac{i}{2^{n}} \chi_{A_{i}} .
\end{aligned}
$$

$f_{n}$ is resolutive and $f_{n} \uparrow f$. Hence $f$ is resolutive. This result can be extended immediately to a Borel function $f,|f| \leq f_{0}$.

Corollary 3. Let $s$ be a positive finite and continuous hyperharmonic function on $X, U$ be an M.P. $P_{0 .-s e t}^{*}$, and $p$ be a locally bounded potential on $U$ positive in a neighbourhood of any boundary point of $U$. Then any Borel function $f,|f| \leq s$, is resolutive.

In this case $A^{*}=\partial U$ and $\bar{H}_{s}$ is harmonic and bounded in the neighbourhood of any boundary point of $U$.

Let $U$ be an open set and $U^{\circ}$ be the set of points $x \in U$ for which any locally bounded potential on $U$ vanishes at $x$. $U^{\circ}$ is closed in $U$. If $U$ is $\sigma^{-}$ compact and $U^{\circ}$ compact, then there exists a locally bounded potential on $U$ positive in the neighbourhood of $\partial U$.

Lemma 4. Let $x \in U$ and $U_{x}$ denote the set of points $y \in U-\{x\}$ such that if $s_{1}, s_{2}$ are locally bounded non-negative hyperharmonic functions on $U$ then

$$
s_{1}(x) s_{2}(y)-s_{1}(y) s_{2}(x)=0 .
$$

If $x \notin X_{0} \cup \bar{U}_{x}$ then $x \notin U^{\circ}$.

Let $\mathscr{C}$ denote the set of restrictions on $U^{\circ}$ of the set of non-negative harmonic functions on $U$. If $f_{1}, f_{2} \in \mathscr{C}$ then $\min \left(f_{1}, f_{2}\right) \in \mathscr{C}$. Indeed let $u_{i}(i=1$, 2 ) be a non-negative harmonic function on $U$ whose restriction on $U^{\circ}$ coincides with $f_{i}$. Then $\min \left(u_{1}, u_{2}\right)$ is a locally bounded non-negative hyperharmonic function on $U$. Denote by $u$ the greatest harmonic minorant of $\min \left(u_{1}, u_{2}\right)$. Since $\min \left(u_{1}, u_{2}\right)-u$ is a potential on $U$

$$
\min \left(f_{1}, f_{2}\right)=\min \left(u_{1}, u_{2}\right)=u
$$

on $U^{\circ}$.

Let $V$ be a regular set in $U, x \in V, \bar{V} \cap U_{x}=\phi, F$ be the carrier of $\omega_{x}^{V}$, and $p$ be a locally bounded potential on $U$. Since $x \notin X_{0}, F \neq \phi$, for a sufficiently small $V$. Suppose $x \in U^{\circ}$. From

*) If $\mathscr{L}$ satisfies Brelot's axioms, the existence of $s$ implies that any open set is an M.P.-set ([3], Part IV, Theorem 3 (ii)). 


$$
0=p(x) \geq \int p d \omega_{x}^{v}
$$

it follows that $p$ vanishes on $F$ and $F \subset U^{\circ}$.

Let $y \in F$ and $s_{1}, s_{2}$ be two locally bounded non-negative hyperharmonic functions on $U$ such that

$$
s_{1}(x) s_{2}(y)-s_{2}(x) s_{1}(y) \neq 0 .
$$

Let $u_{i}(i=1,2)$ be the greatest harmonic minorant of $s_{i}$. Since $s_{i}-u_{i}$ is a locally bounded potential on $U, s_{i}=u_{i}$ on $U^{\circ}$ and

$$
u_{1}(x) u_{2}(y)-u_{2}(x) u_{1}(y) \neq 0 .
$$

If $u_{i}(x)=0$ then from

$$
u_{i}(x)=\int u_{i} d \omega_{x}^{v}
$$

it would result $u_{i}(y)=0$ which contradicts the above inequality. We may suppose therefore

$$
u_{1}(x)=u_{2}(x)=1, \quad u_{1}(y)<u_{2}(y) .
$$

Since $x \in U^{\circ}, F \subset U^{\circ}$,

$$
\int \min \left(u_{1}, u_{2}\right) d \omega_{x}^{v}=\min \left(\int u_{1} d \omega_{x}^{v}, \int u_{2} d \omega_{x}^{v}\right)
$$

and we get the contradictory inequality

$$
0<\int\left(u_{2}-\min \left(u_{1}, u_{2}\right)\right) d \omega_{x}^{r}=u_{2}(x)-\min \left(u_{1}(x), u_{2}(x)\right)=0 .
$$

It follows from this lemma that if $\mathscr{A}$ satisfies Bauer's Trennungsaxiom, then $U^{\circ}$ is empty.

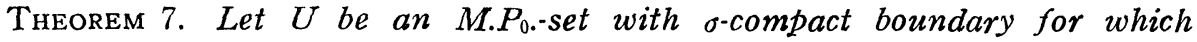
either $\left(\bar{U}^{\circ} \cap \partial U\right) \cup\left(\partial U-A^{*}\right)$ or $\left(\bar{U}^{\circ} \cap \partial U\right) \cup\left(\partial U-A_{*}\right)$ is negligible. If $f$ is a real continuous function on $\partial U$ for which $\bar{H}_{|f|}$ is harmonic and bounded in the neighbourhood of any boundary point of $U$ then $f$ is harmonic resolutive.

It is sufficient to prove this theorem for a non-negative $f$. Replacing $X$ by $X-\{x \in \partial U \mid f(x)=0\}$, we may suppose further $f$ positive. Let $x \in U$ and $s_{x}$ be a non-negative hyperharmonic function finite at $x$ and such that 


$$
\lim _{I I \exists \rightarrow y} \inf _{x \rightarrow y}(z)=\infty
$$

for any $y \in\left(\bar{U}^{\circ} \cap \partial U\right) \cup\left(\partial U-A^{*}\right)$ (resp. $\left.y \in\left(\bar{U}^{\circ} \cap \partial U\right) \cup\left(\partial U-A_{*}\right)\right)$. For any natural number $n$ we denote

$$
A_{n}=\left\{y \in \partial U \mid \lim _{U \ni z \rightarrow y} \inf _{x}(z)>n \max \left(f(y), \lim _{U \ni z \rightarrow y} \sup _{U} \bar{H}_{f}(z)\right)\right\} .
$$

$A_{n}$ is an open set on $\partial U$ which contains the set $\left(\bar{U}^{\circ} \cup \partial U\right) \cup\left(\partial U-A^{*}\right)$ (resp. $\left.\left(\bar{U}^{\circ} \cap \partial U\right) \cup\left(\partial U-A_{*}\right)\right)$.

Let $\varepsilon$ be a positive number. For any $y \in \partial U-A_{n}$ we take a regular neighbourhood $V_{y}$ of $y$ and a hyperharmonic (resp. hypoharmonic) function $s_{y}$ on $U \cap V_{y}$ which satisfy the following conditions: a) $\bar{V}_{y} \cap U^{\circ}=\phi$; b) for any $z \in V_{y} \cap \partial U$ we have $|f(z)-f(y)|<\varepsilon f(z)$; c) $1-\varepsilon<s_{y}<1+\varepsilon$. There exists a compact set $K_{y}$ on $U \cap \partial V_{y}$ and an open set $W_{y}$ on $V_{y} \cap \partial U$ containing $y$ such that

$$
f(y)+\sup _{z^{\prime} \in U \cap^{\prime}{ }^{\prime} y}\left(\bar{H}_{f}\left(z^{\prime}\right)\right) \omega_{z}^{V y}\left(U \cap \partial V_{y}-K_{y}\right)<\varepsilon f(z)
$$

for any $z \in W_{y}$. Since $\partial U-A_{n}$ is $\sigma$-compact there exists a sequence $\left\{y_{i}\right\}$ in $\partial U-A_{n}$ such that

$$
\partial U-A_{n} \subset \bigcup_{i=1}^{\infty} W_{\jmath_{i}}
$$

For any $i$ there exists a potential $p_{i}$ on $U$, finite at $x$ and positive on $K_{y_{i}}$ because $K_{y_{i}} \cap U^{\circ}=\phi$. Let $p_{i x}$ denote the hyperharmonic function associated to $p_{i}$ and $x$ by Lemma 1 , with

$$
p_{i x}(x)<\frac{1}{2^{i}}
$$

and let us denote

$$
p_{x}=\sum_{i=1}^{\infty} p_{i x}
$$

Let $\eta>0, y \in \partial U$ and $\mathfrak{U}$ be an ultrafilter on $U$ converging to $y$ such that

$$
\begin{gathered}
\lim _{\mathfrak{U}}\left(\bar{H}_{f}-\frac{s_{x}}{n}-\eta p_{x}\right)=\lim _{U \ni z \rightarrow y} \sup _{z \rightarrow y}\left(\bar{H}_{f}(z)-\frac{s_{x}(z)}{n}-\eta p_{x}(z)\right) \\
\left(\text { resp. } \lim _{\mathfrak{U}}\left(\underline{H}_{f}+\frac{s_{x}}{n}+\eta p_{x}\right)=\lim _{U \ni z \rightarrow y} \inf \left(\underline{H}_{f}(z)+\frac{s_{x}(z)}{n}+\eta p_{x}(z)\right)\right) .
\end{gathered}
$$

If $y \in A_{n}$ then 


$$
\begin{gathered}
\lim _{\mathfrak{U}}\left(\bar{H}_{f}-\frac{s_{x}}{n}-\eta p_{x}\right) \leq 0 \\
\text { resp. } \left.\lim _{\mathfrak{U}}\left(\underline{H}_{f}+\frac{s_{x}}{n}+\eta p_{x}\right) \geq f(y)\right) .
\end{gathered}
$$

If $y \in A^{*}-A_{n}$ (resp. $y \in A_{*}-A_{n}$ ) then there exists an $i$ such that $y \in W_{y_{i}}$. If $\lim _{\mathfrak{u}} p_{i} \neq 0$

then

$$
\begin{aligned}
& \lim _{\mathfrak{U}} p_{x}=\infty, \\
& \lim _{\mathfrak{U}}\left(\bar{H}_{f}-\frac{s_{x}}{n}-\eta p_{x}\right)=-\infty, \\
& \text { (resp. } \left.\lim _{\mathfrak{U}}\left(\underline{H}_{f}+\frac{s_{x}}{n}+\eta p_{x}\right)=+\infty\right) .
\end{aligned}
$$

If

$$
\lim _{\mathfrak{u}} p_{i}=0
$$

then it can be proved like in Lemma 2 that

$$
\begin{aligned}
& \lim _{\mathfrak{U}} \bar{H}_{f} \leq \theta(\varepsilon) f(y), \quad \theta(\varepsilon)=\left(\frac{1+\varepsilon}{1-\varepsilon}\right)^{2}+\varepsilon \\
& \text { (resp. } \left.\lim _{\mathfrak{u}} \underline{H}_{f} \geq \theta(-\varepsilon) f(y)\right) .
\end{aligned}
$$

For any $y \in \partial U$ we have therefore

$$
\begin{aligned}
& \lim _{U \ni z \rightarrow y} \sup _{f}\left(\bar{H}_{f}(z)-\frac{s_{x}(z)}{n}-\eta p_{x}(z)\right) \leq \theta(\varepsilon) f(y) \\
& \left(\text { resp. } \lim _{\varepsilon^{\prime} \exists z \rightarrow !} \inf _{f}\left(\underline{H}_{f}(z)+\frac{s_{x}(z)}{n}+\eta p_{x}(z)\right) \geq \theta(-\varepsilon) f(y)\right) .
\end{aligned}
$$

If $\left(\bar{U}^{\circ} \cap \partial U\right) \cup\left(\partial U-A_{k}\right)$ is negligible then $\underline{H}_{f}+\frac{s_{x}}{n}+\eta p_{x} \in \overline{\mathscr{S}}_{\theta(-\varepsilon) f}^{U}$ and we get, for a sufficiently small $\varepsilon$,

$$
\underline{H}_{f}+\frac{s_{x}}{n}+\eta p_{x} \geq \bar{H}_{0(-\varepsilon) f}=\theta(-\varepsilon) \bar{H}_{f}
$$

$p_{x}$ and $s_{x}$ being finite at $x$ we get

$$
\underline{H}_{f}(x) \geq \bar{H}_{f}(x)
$$

making successively $\eta \downarrow 0, \varepsilon \downarrow 0, n \uparrow \infty . \quad f$ is therefore resolutive.

Let now $\left(\bar{U}^{\circ} \cap \partial U\right) \cup\left(\partial U-A^{*}\right)$ be negligible and $G$ be an open relatively compact set for which $X_{0} \cap \partial G=\phi$. We denote by $f_{G}$ the function on $\partial(U \cap G)$ equal to 0 on $\bar{G} \cap \partial U$ and equal to $\bar{H}_{f}$ on $U \cap \partial G$ and by $s_{G}$ the function on $U$ equal to $\bar{H}_{f}$ on $U-\bar{G}$ equal to $\bar{H}_{f G}^{G \cap T}$ on $G \cap U$ and equal to 


$$
\lim _{G \cap U \in \inf _{z}} \bar{H}_{f_{G}}^{G \cap U}(z)
$$

for any $z^{\prime} \in U \cap \partial G$. It has been shown in the proof of Lemma 3 that $s_{G}$ is a hyperharmonic function. Let $n$ be a natural number, $\eta^{\prime}$ be a positive number, $y \in G \cap \partial L^{\top}$, and $\mathfrak{H}$ be an ultrafilter on $G \cap U$ converging to $y$ such that

$$
\lim _{\mathfrak{u}}\left(s_{G}-\frac{s_{x}}{n}-\eta^{\prime} p_{x}\right)=\lim _{U \exists z \rightarrow ! j} \sup _{U}\left(s_{G}(z)-\frac{s_{x}(z)}{n}-\eta p_{x}(z)\right) .
$$

If $y \in A_{n}$ then

$$
\lim _{\mathfrak{U}}\left(s_{G}-\frac{s_{x}}{n}\right) \leq 0
$$

If $y \in \partial U-A_{n}$ then there exists an $i$ such that $y \in W_{y_{i}}$. If

$$
\lim _{\mathfrak{u}} p_{i} \neq 0
$$

then

$$
\lim _{\mathfrak{u}}\left(s_{G}-\eta^{\prime} p_{x}\right)=-\infty
$$

If

$$
\lim _{\mathfrak{U}} p_{i}=0
$$

then it can be proved like in Lemma 2 that

$$
\lim _{\mathfrak{U}} \boldsymbol{s}_{G} \leq \varepsilon f(y) .
$$

For any case we get

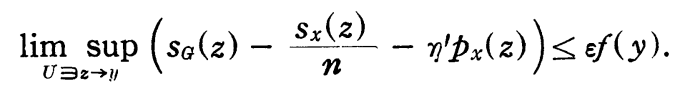

Let us denote by $\&$ the set of relatively compact open sets $G$ for which $X_{0} \cap \partial G=\phi$ and by $u$ the greatest lower bound of the family $\left\{s_{G}\right\}_{G \in \text { S }} . \quad u$ is harmonic. Let $s \in \overline{\mathscr{S}}_{f}^{U}$. Then $s \geq u$ and for any $y \in \partial U$

$$
\liminf _{U \exists z \rightarrow \underline{u}}\left(s(z)-u(z)+\frac{s_{x}(z)}{n}+\eta^{\prime} p_{x}(z)\right) \geq(1-\varepsilon) f(y) .
$$

It follows

$$
s-u+\frac{s_{x}}{n}+\eta^{\prime} p_{x} \geq(1-\varepsilon) \bar{H}_{f}
$$

$p_{x}$ and $s_{x}$ being finite at $x$ we get $u(x)=0$ making succesively $\eta^{\prime} \downarrow 0, \varepsilon \downarrow 0$, $n \uparrow \infty, s(x) \downarrow \bar{H}_{f}(x)$. Let $\left\{G_{i}\right\}$ be a sequence in such that 


$$
s_{0}=\sum_{i=1}^{\infty} s_{G_{i}}
$$

is finite at $x$. Then $\bar{H}_{f}-\eta s_{0}$ is non-positive outside a compact set of $X$. It follows

$$
\begin{aligned}
& \bar{H}_{f}-\frac{s_{x}}{n}-\eta p_{x}-\eta s_{0} \in \underline{\mathscr{S}}_{\theta(\varepsilon) f}^{U}, \\
& \bar{H}_{f}-\frac{s_{x}}{n}-\eta p_{x}-\eta s_{0} \leq \underline{H}_{\theta(\varepsilon) f} .
\end{aligned}
$$

Since $s_{x}, p_{x}, s_{0}$ are finite at $x$ we get

$$
\bar{H}_{f}(x) \leq \underline{H}_{f}(x)
$$

making succesively $\eta \downarrow 0, \varepsilon \downarrow 0, n \uparrow \infty . \quad f$ is therefore resolutive.

If $\mathscr{A}$ satisfies the axioms of Brelot's or Bauer's theory then $X_{0}=U^{\circ}=\phi$ and $A^{*}=A_{*}=\partial U$. Therefore Theorem 7 contains Hervé's [5] and Bauer's [1] (Satz 24) results about the resolutivity of continuous functions on relatively compact open sets. On the other hand, in Brelot's axiomatic there exists always a positive potential on $U$ and the same is true in Bauer's axiomatic if $U$ is $\sigma$ compact. Hence Theorem 6 proves the resolutivity of bounded Borel-measurable functions in these cases. This gives the possibility to prove, without the condition that $X$ has a countable basis, that if $\mathscr{\&}$ satisfies Brelot's axioms and the axiom $D$ [3] the limit of a decreasing sequence of non-negative hyperharmonic functions differs from a hyperharmonic function on a polar set.

The condition $A^{*}=A_{*}=\partial U$ and the fact that the constants are harmonic is not sufficient in order that any continuous function on $\partial U$ is resolutive, even if $U$ is relatively compact. An example is given by a region on a compact Riemann surface, whose boundary consists of more than one point and is of capacity zero.

3. Relation between the normed and the usual Dirichlet problem. The study of the Dirichlet problem on $X$ is interesting only in the case when $X$ is a non-compact M.P.-set. We shall suppose from now on that $X$ satisfies this condition. Let $Y$ be a compactification of $X$, i.e., a compact Hausdorff space which contains $X$ as a dense subspace and $\Delta=Y-X$. Since $X$ is locally compact, $\Delta$ is compact. Let $f$ be a numerical function on $\Delta$. We denote by $\overline{\mathscr{S}}_{f}^{X, Y}=\overline{\mathscr{S}}_{f}^{X}$ (resp. $\mathscr{L}_{f}^{X}, Y=\mathscr{S}_{f}^{X}$ ) the set of lower bounded hyperharmonic (resp. upper bounded. 


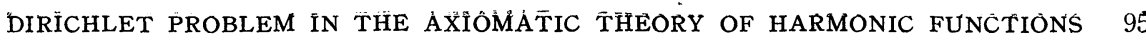

hypoharmonic) functions $s$, such that

$$
\lim _{x \ni x \rightarrow y} \inf _{x \rightarrow y} s(x) \geq f(y) \quad\left(\text { resp. } \lim _{x \ni x \rightarrow y} \sup _{x} s(x) \leq f(y)\right)
$$

for any $y \in 4$. The greatest lower bound of $\overline{\mathscr{S}}_{f}^{x}$ (resp. the least upper bound of $\mathscr{S}_{f}^{X}$ ) is denoted by $\bar{H}_{f}^{X, Y}=\bar{H}_{f}^{X}=\bar{H}_{f}$ (resp. $\underline{H}_{f}^{X}, Y=\underline{H}_{f}^{X}=\underline{H}_{f}$ ); since $X$ is an M.P.-set $\underline{H}_{f} \leq \bar{H}_{f}$. If the functions $\bar{H}_{f}$ and $\underline{H}_{f}$ are finite and equal, the function $f$ is called resolutive and

$$
H_{f}^{X}, Y=H_{f}^{X}=H_{f}=\bar{H}_{f}=\underline{H}_{f}
$$

is called the solution of the Dirichlet problem with $f$ as the boundary function.

If any bounded continuous (resp. lower semi-continous) function on $\Delta$ is resolutive then $Y$ is called a Baire (resp. Borel) resolutive compactification of $\boldsymbol{X}$.

In the rest of this paper $Y$ will be a fixed compactification of $X$.

The normed Dirichlet problem and the Dirichlet problem formulated above are closely related. Indeed let $U$ be an open set for which $\partial U$ is not empty and $f$ be a numerical function defined on $\partial U$. Let $\bar{U}^{Y}$ (resp. $\partial_{Y} U$ ) denote the closure (resp. the boundary) of $U$ in $Y$. Taking $U$ instead of $X$ and $\bar{U}^{Y}$ instead of $Y$ in the preceding considerations, and defining $f_{0}$ equal to $f$ on $\partial_{X} U$ and equal to zero on $\partial_{Y} U-\partial_{X} U$ it is clear that

$$
\overline{\mathscr{P}}_{f}^{U}, x \subset \overline{\mathscr{S}}_{f_{0}}^{U, \bar{U}^{Y}}, \quad \mathscr{\mathscr { P }}_{f}^{U},{ }^{X} \subset \underline{\mathscr{S}}_{f_{0}}^{U}, \bar{U}^{Y} .
$$

Therefore we have

$$
\bar{H}_{f}^{(f, x} \geqq \bar{H}_{f_{0}}^{(j,} \bar{U}^{Y}, \quad \underline{H}_{f}^{(T, x} \leqq \underline{H}_{f_{0}}^{(j,} \bar{U}^{Y} .
$$

If any bounded Borel function on $\partial_{X} U$ is resolutive then $U$ is called a Borelresolutive set.

Lemma 5. Let $Y$ be a compactification of $X, U$ be a Borel resolutive set, and $f$ be a non-negative resolutive function on 4 . Then the function $g$ on $\partial_{Y} U$, equal to $f$ on $\triangle \cap \partial_{Y} U$ and equal to zero on $X \cap \partial_{Y} U=\partial_{X} U$, is resolutive.

We set $u=H_{f}^{X, Y}$. Let $\bar{s}$ (resp. $\underline{s} \geq 0$ ) belong to $\overline{\mathscr{S}}_{f}^{X, Y}$ (resp. $\mathscr{\mathscr { S }}_{f}^{X},{ }^{Y}$ ) and $\underline{s}^{\prime} \geq 0$ (resp. $\left.\bar{s}^{\prime}\right)$ belong to $\underline{S}_{\bar{s}}^{U, X}$ (resp. $\left.\overline{\mathscr{S}}_{s}^{U, x}\right)$. Then $\bar{s}-\underline{s}^{\prime}\left(\right.$ resp. $\left.\underline{s}-\bar{s}^{\prime}\right)$ belongs to $\overline{\mathscr{S}}_{g}^{V,} \bar{U}^{Y}$ (resp. $\mathscr{S}_{g}^{U, \bar{U}^{X}}$ ) and therefore $\bar{H}_{g}^{U, \bar{U}^{Y}}$ is finite and 


$$
\bar{H}_{g}^{U,} \bar{U}^{Y}-\underline{H}_{g}^{U}, \bar{U}^{Y} \leq\left(\bar{s}-\underline{s}^{\prime}\right)-\left(\underline{s}-\bar{s}^{\prime}\right) .
$$

Since $\underline{s}^{\prime}, \bar{s}^{\prime}$ are arbitrary we get

$$
\bar{H}_{g}^{\prime r}, \bar{U}^{Y}-\underline{H}_{g}^{U,} \bar{U}^{Y} \leq\left(\bar{s}-\underline{H}_{s}^{U}, X\right)-\left(\underline{s}-\bar{H}_{\underline{s}}^{I T}, x\right) .
$$

$U$ being a Borel resolutive set and $\underline{s}$ a bounded upper semi-continuous function on $\partial_{\mathrm{Y}} U$ we have

$$
\begin{aligned}
& \bar{H}_{\underline{s}}^{U, X}=\underline{H}_{\underline{s}}^{v, X} \leq \underline{H}_{s}^{U, x}, \\
& \bar{H}_{g}^{U, \vec{U}^{Y}}-\underline{H}_{g}^{U, \bar{U}^{Y}} \leq \bar{s}-\underline{s} .
\end{aligned}
$$

$\bar{s}, \underline{s}$ being arbitrary it follows that $g$ is resolutive.

TheOREM 8. If $Y$ is a Baire (resp. Borel) resolutive compactification of $X$ and $U$ is a Borel resolutive open subset of $X$, then $\bar{U}^{Y}$ is a Baire (resp. Borel) resolutive compactification of $U$.

Since the resolutive functions form a real vector space it is sufficient to prove that any continuous (resp. lower semi-continuous) bounded non-negative function $f^{\prime}$ on $\partial_{Y} U$ is resolutive. Let $f$ be a continuous (resp. lower semicontinuous) bounded non-negative function on $\Delta$ equal to $f^{\prime}$ on $\Delta \cap \bar{U}^{Y}$ and $g$ be equal to $f=f^{\prime}$ on $\Delta \cap \bar{U}^{Y}$ and equal to zero on $\partial_{x} U$. From the preceding lemma it follows that $g$ is resolutive. Since $U$ is Borel resolutive the function $f^{\prime}-g$ is also resolutive, hence $f^{\prime}$ is resolutive.

\section{BIBLIOGRAPHY}

[1] H. Bauer, Axiomatische Behandlung des Dirichletschen Problems für elliptische und parabolische Differentialgleichungen, Math. Ann., 146, (1962), 1-59.

[2] M. Brelot, Axiomatique des fonctions harmoniques et surharmoniques dans un espace localement compact, Séminaire de Théorie du Potentiel, 2 (1959), 1.1-1.40.

[3] M. Brelot, Lectures on Potential Theory, Tata Institute of Fund. Reasearch, Bombay (1960).

[4] M. Brelot, Étude comparée de quelques axiomatiques des fonctions harmoniques et surharmoniques, Séminaire de Théorie du Potentiel, 6 (1962), 1.13-1.26.

[5] R.-M. Hervé, Développements sur une théorie axiomatique des fonctions surharmoniques, Comptes rendus Acad. Sci. (Paris), 248 (1959), 179-181. 